\title{
\begin{tabular}{l|l} 
MitTraries & DSpace@MIT
\end{tabular}
}

\author{
MIT Open Access Articles
}

\section{Quantum Cryptography Approaching the Classical Limit}

The MIT Faculty has made this article openly available. Please share how this access benefits you. Your story matters.

Citation: Weedbrook, Christian et al. "Quantum Cryptography Approaching the Classical Limit." Physical Review Letters 105.11 (2010): 110501. (C) 2010 The American Physical Society

As Published: http://dx.doi.org/10.1103/PhysRevLett.105.110501

Publisher: American Physical Society

Persistent URL: http://hdl.handle.net/1721.1/60865

Version: Final published version: final published article, as it appeared in a journal, conference proceedings, or other formally published context

Terms of Use: Article is made available in accordance with the publisher's policy and may be subject to US copyright law. Please refer to the publisher's site for terms of use. 


\title{
Quantum Cryptography Approaching the Classical Limit
}

\author{
Christian Weedbrook, ${ }^{1,2, *}$ Stefano Pirandola, ${ }^{3}$ Seth Lloyd, ${ }^{2,4}$ and Timothy C. Ralph ${ }^{1}$ \\ ${ }^{1}$ Department of Physics, University of Queensland, St Lucia, Queensland 4072, Australia \\ ${ }^{2}$ Research Laboratory of Electronics, Massachusetts Institute of Technology, Cambridge Massachusetts 02139, USA \\ ${ }^{3}$ Department of Computer Science, University of York, York YO10 5DD, United Kingdom \\ ${ }^{4}$ Department of Mechanical Engineering, Massachusetts Institute of Technology, Cambridge Massachusetts 02139, USA
}

(Received 20 April 2010; revised manuscript received 4 August 2010; published 8 September 2010)

We consider the security of continuous-variable quantum cryptography as we approach the classical limit, i.e., when the unknown preparation noise at the sender's station becomes significantly noisy or thermal (even by as much as $10^{4}$ times greater than the variance of the vacuum mode). We show that, provided the channel transmission losses do not exceed $50 \%$, the security of quantum cryptography is not dependent on the channel transmission, and is therefore incredibly robust against significant amounts of excess preparation noise. We extend these results to consider for the first time quantum cryptography at wavelengths considerably longer than optical and find that regions of security still exist all the way down to the microwave.

DOI: 10.1103/PhysRevLett.105.110501

PACS numbers: 03.67.Dd, 03.67.Hk, 42.50.-p

Introduction.-Quantum key distribution (QKD) using continuous variables (CV) [1,2] allows two people, Alice and Bob, to generate a secure key which can be used to encrypt messages. CV-QKD protocols using Gaussian modulation [3-8], initially begin with Alice preparing a number of randomly displaced pure coherent states and sending them over an insecure quantum channel to Bob. Generally, it is assumed that Alice's states must be pure quantum states to a good approximation otherwise her ability to perform QKD will rapidly become compromised. This seemed to be borne out by recent calculations [9] that showed that the distance over which CV-QKD was secure, when Alice used mixed coherent states in the protocol, fell rapidly as the states became significantly impure.

In this Letter, we show that, provided the channel transmission losses do not exceed $50 \%$, the security of quantum cryptography is not dependent on the channel transmission, and is therefore incredibly robust against significant levels of impurity of Alice's states, without the additional previous requirement of purifiers [9]. This is a remarkable result as we might naturally expect that as Alice's states become more and more thermalized secure transmission over any finite distance would become impossible. This further motivates an investigation of the security of CVQKD as we move from optical frequencies into the infrared and down into the microwave region. As the wavelength gets longer there is no direct way of detecting single photons [10], thus ruling out discrete variable approaches. While CV measurements still apply, state preparation and the quantum channel become thermalized by the significant levels of background radiation that exist for longer wavelengths at room temperature. Here we show that CV-QKD remains, in principle, possible over short distances, well into the infrared and into the microwave regime. This surprising result highlights the possibility of short-range quantum cryptography applications at suboptical frequencies.

Quantum cryptography using Gaussian states.-Typical Gaussian modulated CV-QKD protocols begin with Alice randomly modulating a vacuum state to create a coherent state $|\alpha\rangle$ [11]. This random modulation or displacement $\alpha=Q_{A}+i P_{A}$ contains two independent variables $X_{S} \in$ $\left\{Q_{A}, P_{A}\right\}$ chosen from a two-dimensional Gaussian distribution with variance $V_{S}$ and zero mean. It is these continuous variables that will ultimately be used to construct a secret key between Alice and Bob. Alice then sends a whole ensemble of these randomly displaced pure coherent states to Bob over a quantum channel which is monitored by the eavesdropper, Eve. At the output of the channel, Bob measures the incoming states using either homodyne [4] or heterodyne detection [5].

The initial modes prepared by Alice can be described in the Heisenberg picture as $\hat{X}_{A}=X_{S}+\hat{X}_{0}$ where $X_{S}$ describes the classical signal and $\hat{X}_{0}$ the thermal mode. Here the quadratures $\hat{Q}$ and $\hat{P}$ are defined as $\hat{X}_{A} \in$ $\left\{\hat{Q}_{A}, \hat{P}_{A}\right\}$ and $\hat{X}_{0} \in\left\{\hat{Q}_{0}, \hat{P}_{0}\right\}$. The overall variance $V:=$ $V\left(\hat{X}_{A}\right)$ of Alice's initially prepared mode is given by $V=$ $V_{S}+V_{0}$. We can further decompose the variance of the thermal mode $V_{0}:=V\left(\hat{X}_{0}\right)$ into the variance of the pure vacuum mode (which is normalized to 1 ) and the variance of the unknown preparation noise at Alice's station $\beta$ to give $V_{0}=1+\beta$. Typically, in CV-QKD protocols, we simply have $V=V_{S}+1$, i.e., zero preparation noise $(\beta=0)$. In this Letter, we consider the effect of having nonzero preparation noise on Alice's mode preparation, i.e., $\beta>0$. We assume that this preparation noise cannot be controlled or manipulated by Eve.

In the analysis of CV-QKD protocols, the collective Gaussian attacks [12-14] are the most important. In fact, up to a suitable symmetrization of the protocols [15], these 
attacks bound the most powerful eavesdropping strategy allowed by quantum mechanics [15]. The most general form of a collective Gaussian attack is explicitly described in Ref. [14]. This consists in Eve interacting her (independent) ancilla modes with Alice and Bob's mode for each run of the protocol in such a way to generate a memoryless (one-mode) Gaussian channel. Eve's ancillae are then collected in a quantum memory whose measurement is optimized on Alice and Bob's classical communications [14]. For a practical implementation of the protocols, the most important collective Gaussian attack is the one based on the entangling cloner [16] which is exactly the model considered in our Letter. This consists in Eve perfectly replacing the quantum channel between Alice and Bob with her own quantum channel where the loss is simulated by a beam splitter with transmission $T$ (which ranges in value from 0 to 1 ). She then creates her ancilla modes which are two-mode squeezed states [11] [or commonly known as, Einstein-Podolsky-Rosen (EPR) states], with variance $W$. The modes of the EPR beam can be described by the operators $\hat{E}^{\prime \prime}$ and $\hat{E}$. She keeps one mode of the beam $\hat{E}^{\prime \prime}$ and injects the other mode $\hat{E}$ into the unused port of the beam splitter, resulting in the output mode $\hat{E}^{\prime}$. Eve then collectively detects all modes $\hat{E}^{\prime}$ and $\hat{E}^{\prime \prime}$, gathered from each of the runs of the protocol, in a final coherent measurement. The final stages of the protocol consists in Alice and Bob publicly revealing a subset of their data in order to estimate the channel transmission $T$ and excess channel noise $W$ [2]. We also assume that Alice and Bob (and Eve) know the variance of the unknown preparation noise $\beta$ in order to properly estimate the channel noise as opposed to the sum of the channel noise and the preparation noise. However, the shot to shot displacement due to the excess preparation noise remains unknown to everyone. In the final steps of the protocol, Alice and Bob perform a reconciliation protocol (e.g., see [1]) to correct any errors they might have between them and then finally privacy amplification [2] to reduce Eve's knowledge of the key to a negligible, and safe amount.

Reverse reconciliation.-We begin our analysis by first using the $\mathrm{CV}-\mathrm{QKD}$ protocol known as reverse reconciliation [4], which consists in Alice (and Eve) optimally estimating Bob's measurement outcomes. We note that the previous analysis given in [9] also considered thermal state CV-QKD using reverse reconciliation. However, for completeness, we give the derivation for reverse reconciliation which will be helpful in calculating the direct reconciliation case and for a comparison between the two protocols. The secret key rate $R^{\triangleleft}$ for reverse reconciliation where Bob uses homodyne detection is given by $R^{\triangleleft}:=I\left(X_{A}: X_{B}\right)-I\left(X_{B}: E\right)$. Here $I\left(X_{A}: X_{B}\right)$ is called the mutual information between Alice and Bob and defined in terms of the Shannon (or classical) entropy [17]. The quantum mutual information between Eve and Bob $I\left(X_{B}: E\right)$ is given by the Holevo information [18] and describes the greatest amount of information one can extract from a quantum state [19].
The secret key rate $R^{4}$ can be calculated (see supplementary information [19] for complete derivation) for various values of preparation noise, i.e., $V_{0}=1,10,100$, 1000. The results are plotted in Fig. 1 for a lossy channel (i.e., $W=1$ which corresponds to Eve simply inserting a vacuum state into the unused port of the beam splitter). We see that, as expected, the security is dependent on the channel transmission, and starts deteriorating rapidly as the excess preparation noise is increased. In fact, after only a modest increase in preparation noise (from $V_{0}=1$ to $V_{0}=10$ ), the secure region has shrunk to $T \approx>0.89$.

Direct reconciliation.-We now turn our attention to another CV-QKD scheme known as direct reconciliation [3]. Direct reconciliation was the first protocol to show that one could use Gaussian modulated coherent states to create a secure key. Unlike reverse reconciliation, this protocol is a forward-way scheme where Bob (and Eve) are trying to optimally estimate the values of Alice's initial displacements, or encodings, $Q_{A}$ and $P_{A}$. However, direct reconciliation has the drawback in its inability to create a secret key when the loss is greater than $3 \mathrm{~dB}$. This corresponds to $T<0.5$ and can be intuitively thought of as Eve sharing more common information with Alice than Bob does. Consequently, reverse reconciliation (or postselection [7]) is usually considered the most practical CV-QKD protocol [20]. However, as we will see, despite these shortcomings, direct reconciliation offers a surprising advantage as a potential platform for noise tolerant shortrange QKD.

The secret key rate $R^{\nabla}$ for direct reconciliation using homodyne detection is defined as $R^{\triangleright}:=I\left(X_{A}: X_{B}\right)$ $-I\left(X_{A}: E\right)$, where $I\left(X_{A}: E\right)$ is again the Holevo quantity but now defined between Eve and Alice. We can now calculate the subsequent key rates (see supplementary material [19] for details). In Fig. 2 we have plotted the resulting secret key rates for various values of $V_{0}$ using

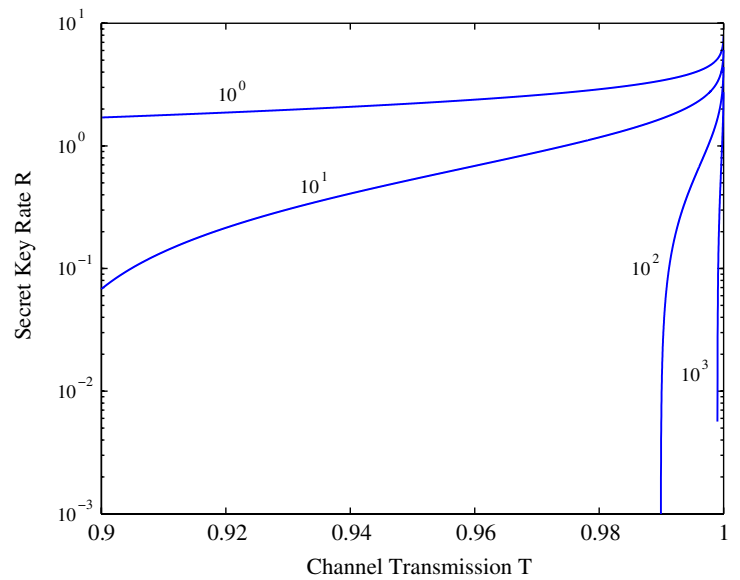

FIG. 1 (color online). Secret key rate $R^{4}$ versus channel transmission $T$ using reverse reconciliation. Increasing the amount of unknown classical noise on Alice's preparation modes in $\mathrm{CV}-\mathrm{QKD}$. Here the thermal radiation is increased: $V_{0}=1,10$, $10^{2}, 10^{3}$ from left to right, where $W=1$ (lossy channel), $V_{S}=$ $10^{5}$, and $V_{0}=1$ is a pure vacuum mode. 


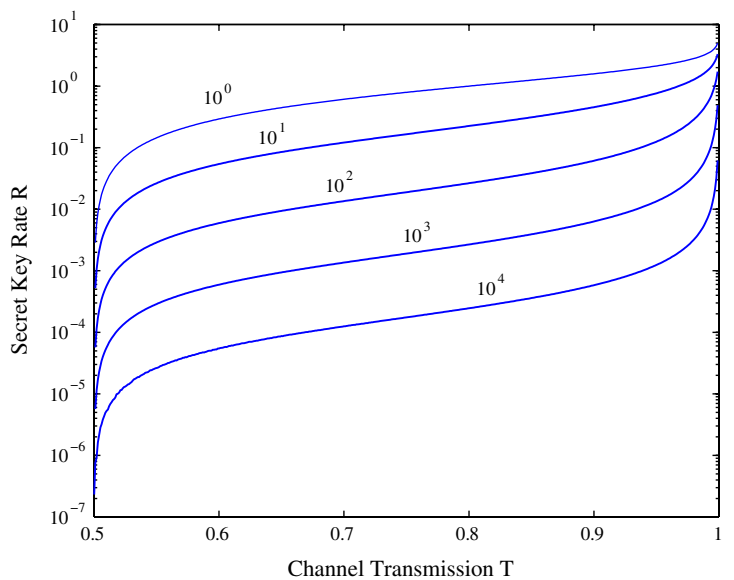

FIG. 2 (color online). Secret key rate $R^{\nabla}$ versus channel transmission $T$ using direct reconciliation. Increasing the amount of unknown classical noise on Alice's preparation modes in $\mathrm{CV}-\mathrm{QKD}$. Here the thermal radiation is increased: $V_{0}=1,10$, $10^{2}, 10^{3}, 10^{4}$ from top to bottom, where $W=1, V_{S}=10^{5}$. We find that direct reconciliation does not show any deterioration in channel loss when excessively large amounts of preparation noise is added.

$W=1$ and $V_{S}=10^{5}$. We find that direct reconciliation has the amazing feature that as the preparation noise becomes more and more significant (even up to $10^{4}$ times that of the variance of the pure vacuum mode) only the secret key rate decreases and not the channel transmission. So for any value of preparation noise the initial starting point is always $T=0.5$ (cf. reverse reconciliation where modest increases in noise reduce the secure region close to unity transmission, i.e., see Fig. 1). The basic physics is that, for $T>0.5$, the presence of quantum noise always gives Alice and Bob a direct information advantage over Eve. Increased preparation noise reduces this advantage, but it always remains finite. In contrast, for reverse reconciliation, Alice's ability to estimate what Bob received is rapidly compromised by the preparation noise. This removes their information advantage over Eve.

In Fig. 3 we have a security threshold plot for direct and reverse reconciliation for $W=1$. The solid (blue) curve is the previous best bound derived using reverse reconciliation and is given by [9]: $\beta<(1-T)^{-1}$. On the same plot we have the new direct reconciliation bound which shows a substantial improvement over the previous reverse reconciliation bound. Remarkably, we can see how direct reconciliation is unaffected by the channel transmission once $T>0.5$ and is secure for a minimum of 4 orders of magnitude of preparation noise. Therefore, it is best to use reverse reconciliation when $T \leq 0.5$ and direct reconciliation when $T>0.5$. Additionally, this result is robust to the addition of small amounts of excess noise on the quantum channel (i.e., $W>1$ ) which moves the transmission limit slightly over $50 \%$ but retains qualitatively the same behavior as the lossy case [21].

Infrared to microwave quantum cryptography.-It is interesting to consider a possible application of our results:



FIG. 3 (color online). Tolerable preparation (classical) excess noise $\beta=V_{0}-1$ versus channel transmission $T$ for direct and reverse reconciliation over a lossy channel. The area under the solid (blue) curve indicates the previous best secure region threshold using reverse reconciliation [9]. However, for direct reconciliation, after $T=0.5$, one can immediately obtain many orders of magnitude improvement in the security threshold.

wireless $\mathrm{CV}-\mathrm{QKD}$ at infrared to microwave frequencies. Today, a large number of popular wireless communication technologies rely on such frequencies to distribute information. Because of the ubiquitous nature of such devices, their security is of fundamental importance. Moving to frequencies lower than optical rules out discrete variable QKD because of the lack of photon counting capabilities. The problem for CV-QKD is that operating at lower frequencies at room temperature inevitably introduces a significant amount of thermal noise. In contrast to the previous section, we now consider a simplified wireless communication protocol where both Alice's preparation modes and the quantum channel (Eve) are affected by the thermal background. When considering Eve we assume that she prepares her attack within a cryostat which allows her to essentially prepare pure modes away from the effect of the background radiation. Then to cover her tracks she adds known excess noise to her pure states to emulate the thermal noise of the environment.

In the previous section we showed that direct reconciliation is significantly more robust against preparation noise than reverse reconciliation and is consequently better suited to our current analysis. Given that, the next step is to calculate how strong the thermal modes are at particular frequencies from optical down to the microwave $[1 \mathrm{GHz}$ $(\lambda=30 \mathrm{~cm})$ to $300 \mathrm{GHz}(\lambda=1 \mathrm{~mm})]$. To do this we first write the average photon number $\bar{n}$ in terms of the quadrature variance $V$ using $\bar{n}=\left\langle\hat{a}^{\dagger} \hat{a}\right\rangle=(V-1) / 2 \Rightarrow V=$ $2 \bar{n}+1$ where we have symmetrized both quadratures, i.e., $V:=V(\hat{Q})=V(\hat{P})$ and the annihilation operator $\hat{a}$ is defined as $\hat{a}=(\hat{Q}+i \hat{P}) / 2$. Second, the average photon number is equal to $\bar{n}=\left[\exp \left(\hbar \omega / k_{B} T\right)-1\right]^{-1}$ [11] and represents the blackbody radiation spectrum. For example, at room temperature $T=300 \mathrm{~K}$ and using a microwave 


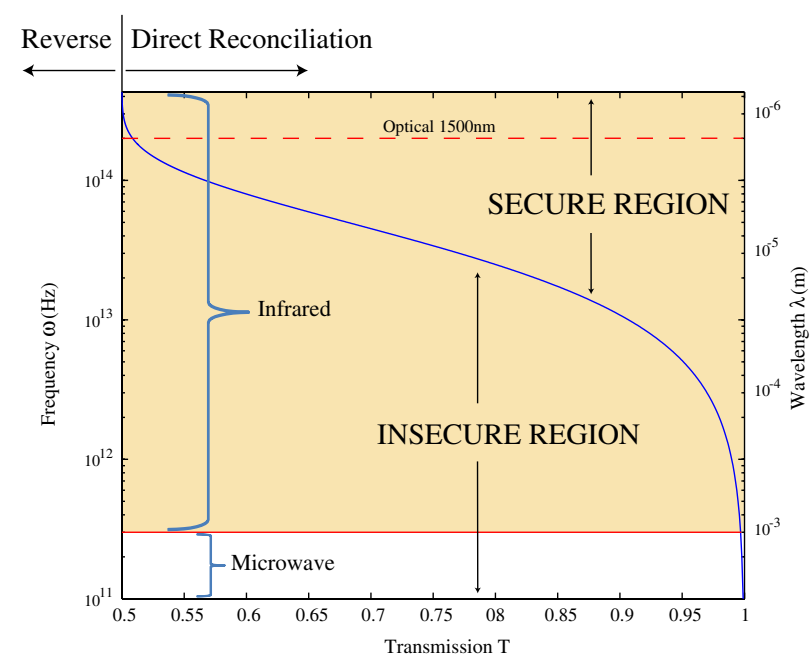

FIG. 4 (color online). Security of quantum cryptography over various electromagnetic wave frequencies (at room temperature) as a function of channel transmission. Moving our way from the infrared spectrum $(430 \mathrm{THz})$ and into the microwave spectrum (300 GHz). Our results show that direct reconciliation should be used when channel losses are less than $50 \%$ and reverse reconciliation otherwise. We note that at each point the same impurity applies to both Alice and Eve with $V_{S}=10^{8}$.

frequency of $\omega=1 \mathrm{GHz}$ we find that the variance of the thermal mode is $V=7.85 \times 10^{4}$; while at the other end of the microwave spectrum $(\omega=300 \mathrm{GH} z)$ the variance is $V=2.63 \times 10^{2}$.

Using the analysis from the previous section, we can calculate the secret key rates using direct reconciliation. In Fig. 4 we plot the security of CV-QKD from the optical frequency $(1500 \mathrm{~nm})$ into the infrared region and down into the microwave frequency as a function of channel line transmission. We point out that the secure region corresponds to $R>0$. We find a window of security for CVQKD throughout all of the infrared region and into the microwave frequency albeit with smaller allowed levels of loss. In the midinfrared region transmission of $T \approx 0.8$ is required while in the case of the microwave region we see that a secure key can only be generated when the transmission is higher than $T \approx 0.9969$. Nonetheless, it is interesting that a small security window, in principle, exists. Future analysis will look at improving the region where infrared and microwave CV-QKD is secure. For example, in [6] they showed that the security thresholds for direct reconciliation could be improved (and in fact beat the $3 \mathrm{~dB}$ loss limit) if two-way quantum communication was used. Furthermore, postselection [7] could also be used to investigate a possible way to combat the high preparation noise.

Conclusion.-In conclusion, we have shown that when considering unknown preparation noise in continuousvariable $\mathrm{QKD}$, direct reconciliation is significantly more robust than reverse reconciliation when the channel loss does not exceed 50\%. Incredibly, direct reconciliation showed no deterioration in the loss threshold, only in secret key rates, even when the variance of the thermal noise is as much as $10^{4}$ times greater than that of the pure vacuum mode. Furthermore, we have shown that infrared to microwave quantum cryptography is, in principle, possible over short distances when using continuous variables and opens up the possibility of further avenues of investigations.

C.W. and T.R. would like to thank the Australian Research Council (ARC) for funding. S. P. was supported by a Marie Curie Action of the European Community. S. L. and C. W. would like to thank the W. M. Keck Foundation Center for Extreme Quantum Information Theory for funding. C. W. would also like to thank Vladyslav Usenko, Radim Filip, Warwick Bowen, Nathan Walk, Denis Sych, Franco Wong, and Jeff Shapiro for helpful discussions.

*christian.weedbrook@gmail.com

[1] N. J. Cerf and Ph. Grangier, J. Opt. Soc. Am. B 24, 324 (2007).

[2] V. Scarani, H. Bechmann-Pasquinucci, N. J. Cerf, M. Dusek, N. Lutkenhaus, and M. Peev, Rev. Mod. Phys. 81, 1301 (2009).

[3] F. Grosshans and P. Grangier, Phys. Rev. Lett. 88, 057902 (2002).

[4] F. Grosshans, G. Assche, J. Wenger, R. Brouri, N. J. Cerf, and P. Grangier, Nature (London) 421, 238 (2003).

[5] C. Weedbrook, A. M. Lance, W. P. Bowen, T. Symul, T. C. Ralph, and P. K. Lam, Phys. Rev. Lett. 93, 170504 (2004).

[6] S. Pirandola, S. Mancini, S. Lloyd, and S. L. Braunstein, Nature Phys. 4, 726 (2008).

[7] C. Silberhorn, T. C. Ralph, N. Lutkenhaus, and G. Leuchs, Phys. Rev. Lett. 89, 167901 (2002).

[8] A. M. Lance, T. Symul, V. Sharma, C. Weedbrook, T. C. Ralph, and P. K. Lam, Phys. Rev. Lett. 95, 180503 (2005).

[9] R. Filip, Phys. Rev. A 77, 022310 (2008); V. C. Usenko and R. Filip, Phys. Rev. A 81, 022318 (2010).

[10] G. Temporão et al., Opt. Lett. 31, 1094 (2006).

[11] C.C. Gerry and P.L. Knight, Introductory Quantum Optics (Cambridge, Cambridge, 2005).

[12] M. Navascués, F. Grosshans, and A. Acín, Phys. Rev. Lett. 97, 190502 (2006).

[13] R. García-Patrón and N. J. Cerf, Phys. Rev. Lett. 97, 190503 (2006).

[14] S. Pirandola, S. L. Braunstein, and S. Lloyd, Phys. Rev. Lett. 101, 200504 (2008).

[15] R. Renner and J. I. Cirac, Phys. Rev. Lett. 102, 110504 (2009).

[16] F. Grosshans, N. J. Cerf, J. Wenger, R. Tualle-Brouri, and Ph. Grangier, Quantum Inf. Comput. 3, 535 (2003).

[17] C.E. Shannon, Bell Syst. Tech. J. 27, 379 (1948).

[18] A. S. Holevo, Probl. Inf. Transm. 9, 177 (1973).

[19] See supplementary material at http://link.aps.org/ supplemental/10.1103/PhysRevLett.105.110501 for a PDF file that contains additional details.

[20] A. Leverrier and Ph. Grangier, Phys. Rev. Lett. 102, 180504 (2009).

[21] C. Weedbrook et al. (to be published). 